\title{
Using Satellite Image, Recognize and detect the vehicles in Digital Image Processing by applying Otsu Threshold Method
}

\author{
Mohd Ashraf, Md. Zair Hussain
}

\begin{abstract}
DIP) is known as a process which uses several computer algorithms. Image processing is done on digital images by using these algorithms. Digital image processing is using in several applications, like image-sharpening, medical, pattern reorganization, color processing, remote sensing, video processing etc. The traffic data is affected by satellite images object oriented detection approach and satellite resolution. As compared with the conventional data gathering approach when data is gathering from satellite images then it can be process more quickly and efficiently. The research works is done for detecting and recognize the vehicle in satellite images. The threshold technique that using in this research is Otsu method. The main objective for this approach to find a more improved and effective approach to detect the vehicles in less time.
\end{abstract}

Index Terms: digital image processing; satellite; vehicle; threshold, otsu.

\section{INTRODUCTION}

The detection of vehicles from the images taken from satellite is an efficient method for recognizing the vehicles. Because the vehicles are recognized form high resolution satellite images. There is much scope for developing an accurate frame work in the field of vehicle detection through satellite image because $100 \%$ accurate system design is very tough task [1].

Vehicle detection and recognition strategy oversees requesting vehicle into a particular class or gathering where article recognition approach binding a specific vehicle of need in moving video or advanced modernized pictures. Everything or article class has its own particular features depict themselves and separate them from the others. along these lines it can recognize the identical or relative articles in various pictures or chronicles. There are numerous application are exist in which article acknowledgment and discovery is connected some of them are computerized vehicle leaving frameworks, picture recovery, security, machine review, observation and so on item acknowledgment recognition still concern numerous Critical troubles. One principle issue is about power with respect to assortment in scale, non-inflexible misshapenings, imaging conditions and perspective. Enormous scale picture recovery is another present issue in this field. Since there should scale up to thousands thing classes and countless pictures [2].

In the image field article are contained and it very well may be perceived normally. We can realize this as item location and this is the serious issue and undertaking in the PC field today.

\section{FUNDAMENTAL OF IMAGE PROCESSING}

In early 1920 in a paper industry image processing was first presented for submarine link move the picture were coded and at the accepting point by a transmit printer picture was imitated. There had been improvements in the system in the mid to late 1920 s. image processing was used to improve the photos of the moon taken by the Ranger 7 space test in 1964. Such systems were used in the other space missions moreover. Picture handling system utilized in the restorative field in 1970s. Allan M. Cormack and Godfrey N. Hounsfield commonly got The Nobel Prize for the production of PC helped tomography in 1979. Presently in nowadays picture handling is getting progressively more thought because of the accentuation on two fundamental areas [3]:

1. To manage image and For self decision observation

2. in the image data for human enhancement and analysis

Analog and digital image processing are two types of digital image processing.

The image processing in which simple or printed copies are required for human survey then analog image processing is required. Printout and photos are the example of analog image processing.

In digital image processing handling by utilizing PC or some other gadgets controls the image in digitized way. Primarily there incorporate three stages in computerized image processing [3]. The stages are appeared in figure 1:

- Low-level image processing

- Mid-level image processing

- High-level image processing

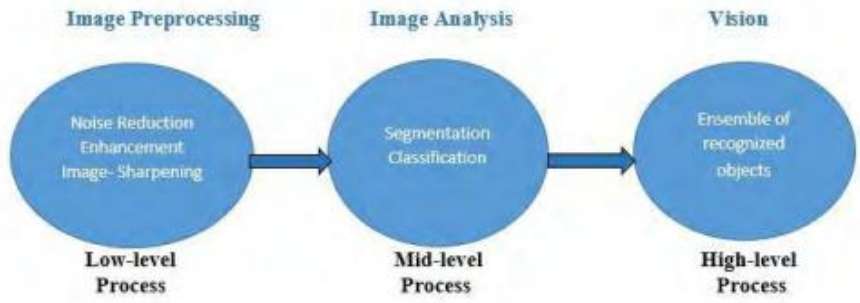

Figure 1: phases of Digital Image Processing

The basic arctitecure of digital image procesing is shown in figure 2 .

Published By:

Blue Eyes Intelligence Engineering
Mohd Ashraf, ${ }^{1}$ Associate Professor, CSE, School of Technology, Maulana Azad National Urdu University, Hyderabad

Md. Zair Hussain, Associate Professor, Information Technology,School of Technology, Maulana Azad National Urdu University, Hyderabad 


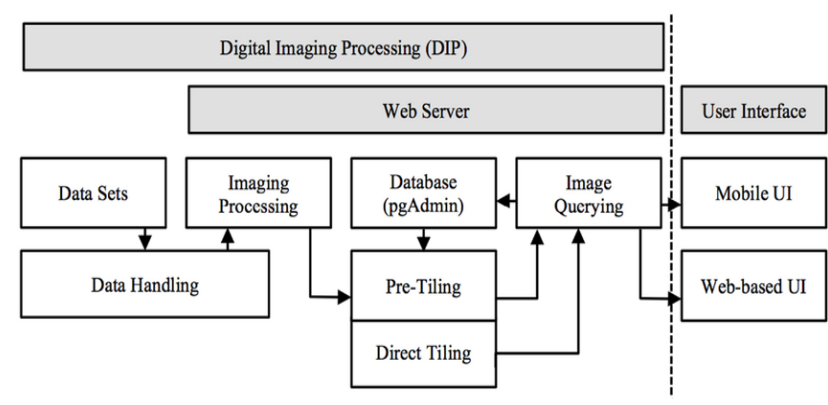

Figure 2: Digital Image Processing architecture

\section{Image Processing TechniQues}

There are several image processing technique techniques are available. This section control formation, processing and acquisition of images. The figure 3 shows the process that included and used for evolution.

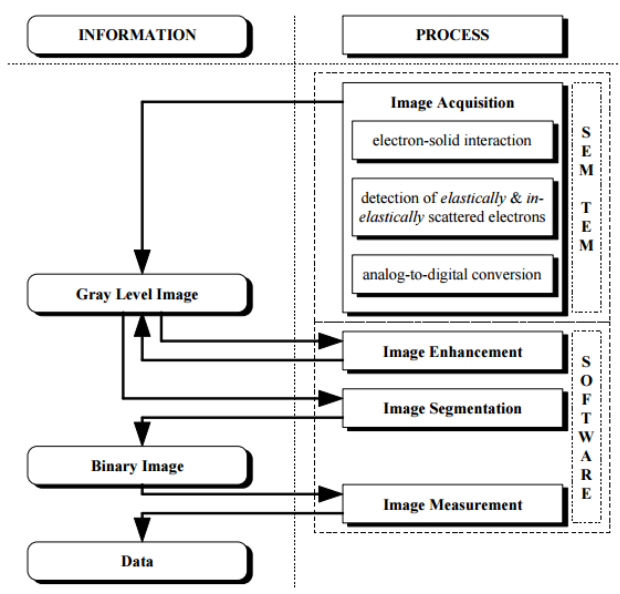

Figure 3: Image processing technique [3]

\section{RELATED WORK}

it is need to detect the change in satellite imagery by using Semantic analysis [4]. This is basically depends on background modeling. To given a brand new image many images have been tested and detected how the normal background looks of the scene. the pixel that have abnormal look considered as change in the object [5].

\section{Challenges of ObJect Detection and Tracking in VIDEO}

In vehicle detection general sense includes evaluating the region of a particular part in dynamic housings in a video gathering or image. Distinguish an vehicle effectively is an exceptionally diligent work, particularly when the object consistently change its shape, size region and heading or article have a confounded structure. In the previous couple of decades to discover the article in a particular video outline there are numerous techniques and calculations have been presented by numerous researchers. Be that as it may, every technique or calculation has their very own detriments and inclinations. Any vehicle identification calculation has their mistakes and that can cause a float. So there is need of a superior calculation that can diminish the float to recognize the precise item over the time distribution of the application.

\section{PROPOSED APPROACH}

It is very important to develop a system which can which can track the vehicle in traffic. In satellite image the object oriented detection and satellite resolution improvement affect the data of traffic.

a. Enhancement of images

Due to the obstruction of some external factors like weather, shadow, chaos and chaos the detection of vehicles from the satellite image is very difficult task. So before extract the vehicles from satellite images to enhance the rate of vehicle detection and precision of the detection it is require to enhance the satellite images acquisition.

b. Grey scale manipulation

In the input image on a $1 \mathrm{x} 1$ pixel neighborhood when the operator ' $\mathrm{T}$ ' acts, at $(\mathrm{x}, \mathrm{y})$ it depend on the value of the ' $\mathrm{F}$ '. This is known as mapping or grey scale transformation.

c. Histogram Equalization

For enhancing the image display histogram equalizer are used. If a taken image is very dark then its histogram alter towards the lower end of the grey scale and in the dark end of the histogram, the detail of image squeezed.

d. Smoothing of images and Segmentation

To reduce the effects of missing pixel values, spurious pixel values and camera noise the smoothing of image is used. edge-preserving smoothing and neighbourhood averaging is techniques are used for smoothing purpose. In the image segmentation image the features that sharing common characteristics are grouped together. This step use region detection, thresholding, edge detection, statistical classification or any combination of these methods.
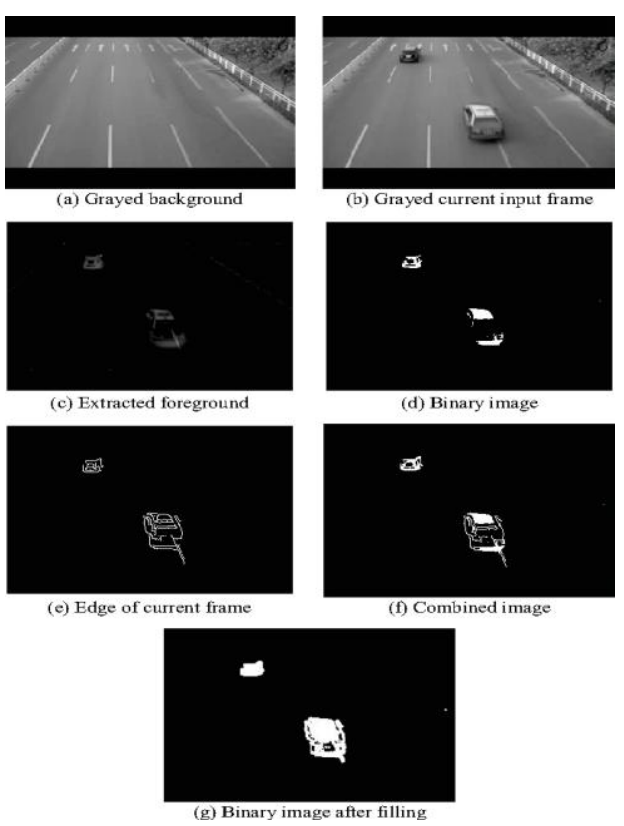

Figure 3: Process for detecting vehicle from an satellite image 


\section{OTSU THREShOLD}

To find the optimal threshold value $\mathrm{k} *$ the Otsu threshold [11] uses the class reparability. It amplifies the between-class change. To extract the object from its background Otsu method is used. For evaluating the method of Otsu threshold $\mathrm{k}^{*}$ MATLAB has a built-in function. On testing image by applying Otsu threshold directly it can detect the bright vehicles. There is necessary to first apply a preprocess step to reduce the problem of road dividers and lane markers on highway.

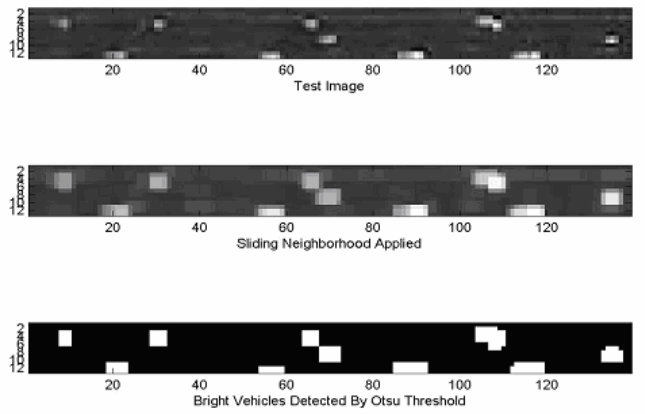

Figure 4: Detection of the Bright Vehicles Using Otsu Threshold

On the testing image the sliding neighborhood operation is used. And in testing image it assign maximum level of intensity of its neighborhood to each pixel. from the large objects the bright By applying sliding neighborhood operation pixels corresponding. The preprocessing step help to highlight the vehicles, and dim the lane markers. To generate a binary image the Otsu Threshold is computed, after applying the sliding neighborhood operation.

\section{Detection of Dark Vehicles}

Otsu threshold is used for detecting the dark vehicles. In a rectangular neighborhood of a 3-by-3 matrix for detecting the dark vehicles minimum intensity is assigned to each pixel. When compared to background the dark vehicle become more darkens. To convert the image in a binary image Otsu threshold is used.
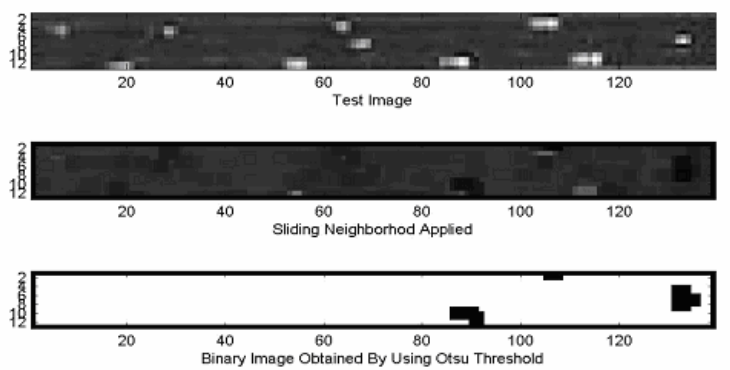

Figure 5: Dark vehicles Detected by Otsu Threshold

VIII. RESUlT

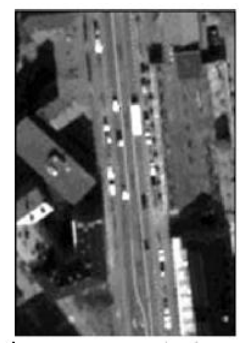

Figure 6: Reference image1

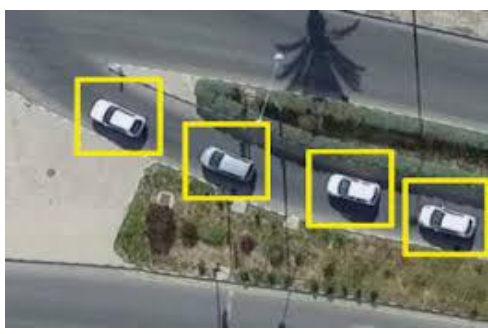

Figure 7: refernce image 4

\begin{tabular}{|c|c|c|}
\hline \multirow[b]{2}{*}{$\begin{array}{l}\text { Reference } \\
\text { Images }\end{array}$} & \multicolumn{2}{|c|}{ Result in \% } \\
\hline & $\begin{array}{l}\text { No of } \\
\text { vehicles }\end{array}$ & Accuracy \\
\hline Image 1 & 11 Vehicles & $84 \%$ \\
\hline Image 2 & 15 Vehicles & $65 \%$ \\
\hline Image 3 & 22 Vehicles & $85 \%$ \\
\hline Image 4 & 4 Vehicles & 1005 \\
\hline
\end{tabular}

Table 1: Result in percentage

\section{Conclusion}

The traffic information is influenced by satellite images object oriented detection and satellite goals. As contrasted and the regular information social affair approach when information is gathering from satellite image then it very well may be process all the more rapidly and productively. The exploration works is accomplished for distinguishing and perceive the vehicle in satellite pictures. The limit strategy that utilizing in this exploration is Otsu technique. The principle objective for this way to deal with locates an increasingly improved and viable way to deal with identify the vehicles in less time. The identification of vehicles from the pictures taken from satellite is an effective technique for perceiving the vehicles. Since the vehicles are perceived structure high goals satellite pictures. There is much scope for building up an exact casing work in the field of vehicle recognition through satellite image in light of the fact that $100 \%$ precise framework configuration is extreme undertaking.

\section{REFERENCES}

1. Noorpreet Kaur Gill,Anand Sharma, "Vehicle Detection from Satellite Images in Digital Image Processing", International Journal of Computational Intelligence Research, ISSN 0973-1873 Volume 13, Number 5 (2017), pp. 697-705.

2. R. Obulakonda Reddy, K.Reddy Madhavi, V. Nagalakshmi, "Moving Objects Detection \& Recognition using Hybrid Canny Edge Detection Algorithm in Digital Image Processing", International Journal of Innovative Technology and Exploring Engineering (IJITEE), Volume-8 Issue-9S3, July 2019.

3. http://www.ncsa.illinois.edu/People/kindr/phd/PART1.PDF

4. K. Sumithra, S. Buvana, R. Somasundaram, "A Survey on Various Types of Image Processing Technique", International Journal of Engineering Research \& Technology (IJERT) Vol. 4 Issue 03, March-2015

5. Andres Huertas and Ramakant Nevatia, "Detecting changes in aerial views of man-made structures," in Proc. of International Conference on Computer Vision, 1998.

6. Aaron J Heller, Yvan G Leclerc, and Quang-Tuan Luong, "A framework for robust 3-d change detection," in Proceedings for International Symposium on Remote Sensing, SPIE., 2001.

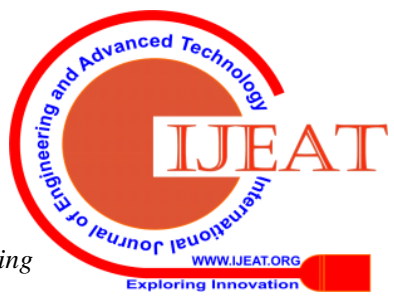

